\title{
ARTICLE
}

\section{An acorn squash (Cucurbita pepo ssp. ovifera) fruit and seed transcriptome as a resource for the study of fruit traits in Cucurbita}

\author{
Lindsay E Wyatt ${ }^{1}$, Susan R Strickler ${ }^{2}$, Lukas A Mueller ${ }^{2}$ and Michael Mazourek ${ }^{1}$
}

Acorn squash (Cucurbita pepo) is an iconic fall vegetable in the United States, known for its unique fruit shape and also prized for its culinary properties. Little is known about the metabolism that underlies the development of fruit quality attributes such as color, sweetness, texture and nutritional qualities in acorn squash, or any other winter squash grown worldwide. To provide insight into winter squash fruit and seed development and add to the genomic resources in the Cucurbita genus, RNA sequencing was used to generate an acorn squash fruit and seed transcriptome from the cultivar Sweet REBA at critical points throughout fruit development. 141838600 high-quality paired-end Illumina reads were assembled into 55949 unigenes. 85\% of unigenes with predicted open reading frames had homology with previously identified genes and over $62 \%$ could be functionally annotated. Comparison with the watermelon and cucumber genomes provided confirmation that the unigenes are full-length and comprehensive, covering an average of $90 \%$ of the coding sequence of their homologs and $72 \%$ of the cucumber and watermelon exomes. Key candidate genes associated with carotenoid and carbohydrate metabolism were identified toward a resource for winter squash fruit quality trait dissection. This transcriptome represents a major advance in C. pepo genomics, providing significant new sequence information and revealing the repertoire of genes expressed throughout winter squash fruit and seed development. Future studies on the genetic basis of fruit quality and future breeding efforts will be enhanced by tools and insights developed from this resource.

Horticulture Research (2015) 2, 14070; doi:10.1038/hortres.2014.70; Published online: 28 January 2015

\section{INTRODUCTION}

Winter squash (Cucurbita spp.) is an important vegetable crop known for its nutritional content and long storage life. It is eaten as a fresh market crop, processed to make frozen squash and canned pumpkin, and the seeds are consumed as a snack food and pressed for edible oil. Winter squash is an excellent source of nutrients, including carotenoids, ascorbic acid and vitamin $C^{1}{ }^{1}$ It is most notable as a source of carotenoids, primarily $\beta$-carotene and lutein, ${ }^{2}$ which are beneficial as a pro-vitamin A compound and for eye health respectively. ${ }^{3}$ Additionally, squash seeds provide protein, lipids, tocopherols and phytosterols. ${ }^{4-6}$ Seeds were likely one of the oldest food uses of this crop, as many of the wild accessions have bitter, inedible pericarps. ${ }^{7}$ Winter squash is a unique source of these nutrients because it can be stored and consumed for many months past the growing season.

To increase the consumption of winter squash, culinary quality is a vital target of improvement through breeding. Fruit quality is determined by several characteristics including color, nutrient content, sweetness, flavor and texture. Color and nutritional value are dually controlled by carotenoid content, ${ }^{8,9}$ with a deeper yellow or orange color and a higher nutrient level seen as desirable. Sweetness is associated with sugar content, and higher sweetness is desirable for consumer acceptance and contributes to improved squash flavor. ${ }^{10}$ Winter squash fruit texture is highly correlated with starch content and dry matter, with higher starch content linked to a superior texture. ${ }^{10-12}$ These quality traits are quantitative and have a complex inheritance, ${ }^{13,14}$ yet are controlled by well-understood metabolic pathways. Understanding the genetic basis of these quality traits will allow for more insight into the breeding of squash for improved fruit quality.

Cucurbita species have a small but growing number of genomic resources that can be employed when studying these quality traits. Other members of the Cucurbitaceae family, namely, cucumber, watermelon and melon, have more extensive genomic resources, including sequenced genomes and many molecular markers. ${ }^{15-21}$ Cucurbita species are diploid $(2 n=2 x=40)$ and have a genome size of approximately $500 \mathrm{Mb}^{22}$ Their small and numerous chromosomes are a remnant of a polyploidy event with persisting duplications $^{23}$ that complicates their molecular breeding. Nevertheless, several genomic maps have been generated for squash ${ }^{24-26}$ including that of Esteras et al., ${ }^{27}$ which was the first squash map to use single nucleotide polymorphism markers. Another major source of genomic information is a C. pepo transcriptome, ${ }^{28}$ comprised of 49 610 unigenes derived from flower, leaf and root tissue. More than $60 \%$ of the unigenes were annotated, aiding in the potential identification of genes of interest in the squash genome. Furthermore, the transcriptome was used to identify more than 10000 potential simple sequence repeats and single nucleotide polymorphisms that could be used to generate molecular markers. In addition, a C. moschata transcriptome was sequenced from leaf, stem, and shoot tissue and assembled into 62480 unigenes. ${ }^{29}$ Sixty-eight percent of the unigenes were annotated and almost 8000 potential simple sequence repeats were identified.

Transcriptome generation through RNA sequencing is a technology that can be used in the dissection of complex traits; fruit transcriptome analysis has been used to successfully identify transcripts involved in early fruit development of cucumber. ${ }^{30,31}$ Assembled

${ }^{1}$ Department of Plant Breeding and Genetics, Cornell University, Ithaca, NY, USA and ${ }^{2}$ Boyce Thompson Institute for Plant Research, Tower Road, Ithaca, NY, USA

Correspondence: M Mazourek (mm284@cornell.edu)

Received: 20 October 2014; Revised: 18 December 2014; Accepted: 22 December 2014 
transcriptomes also provide valuable sequence resources in species lacking a sequenced genome. However, one limitation of RNAsequencing data is that it is specific to the plant line, tissue, developmental stage and physiological condition sequenced. For this reason, it is essential to use transcriptome data relevant to the experimental question of interest. The first $C$. pepo transcriptome, ${ }^{28}$ assembled from summer squash root, leaf and flower tissue, constituted a huge advance in C. pepo genomics. To study aspects of fruit quality, however, knowledge of fruit-specific genes is important. Fruit development and ripening is a process often associated with the expression of suites of genes at defined stages. ${ }^{32,33} \mathrm{~A}$ comprehensive squash fruit transcriptome must therefore sample these specific tissues and stages.

To create a resource for the study of fruit ripening and development in winter squash, in this study, we sequenced and assembled the first Cucurbita pepo fruit and seed transcriptome from the acorn squash cultivar Sweet REBA. Acorn squash is a popular winter squash known for its unique ribbed shape. Of the diverse squash types in C. pepo, the best-characterized Cucurbita species, acorn squash is the longest-storing and has the highest fruit quality. 'Sweet REBA' in particular is an inbred line that is agriculturally successful as a cultivar, making it an ideal candidate for generating a transcriptome that can both build off of the existing C. pepo knowledge and be directly applicable to squash breeding. The transcriptome was generated from five different time points throughout fruit development and derived from both mesocarp and seed tissues. The raw sequence data were assembled into 55 949 unigenes, which were then functionally annotated and compared to the cucumber and watermelon genomes. In addition, putative fruit quality-related transcripts were identified.

\section{MATERIALS AND METHODS}

Plant material

'Sweet REBA', an acorn squash inbred cultivar (M. Jahn, Cornell University), was grown in the Guterman greenhouse facility at Cornell University, Ithaca, NY, USA using standard horticultural practices. Flowers were manually selfpollinated and three representative, randomly-selected fruits per time point were collected at 5, 10,15, 20 and 40 days after pollination. Time points were selected to represent a range of squash fruit and seed developmental stages, including early fruit and seed development ( 5 and 10 days), seed coat formation (15 and 20 days) and fruit ripening, up until the typical harvest date (40 days). ${ }^{34}$ Fruit mesocarp and seed tissues were dissected, flash frozen in liquid nitrogen and stored at $-80{ }^{\circ} \mathrm{C}$ until RNA extraction.

\section{RNA extraction and sequencing}

RNA was extracted from individual fruit and seed samples using the RNeasy Plant Mini Kit (Qiagen, Valencia, CA, USA). Equal amounts of total RNA were pooled together into three independent biological samples, each consisting of RNA from both fruit and seed tissue at all five time points. Library preparation and sequencing were performed by the Genomics Core Facility, Cornell University. The sequencing library was prepared using the TruSeq RNA Sample Preparation Kit v2 (Illumina, San Diego, CA, USA) and pairedend reads were sequenced from the three independent samples on one lane of an Illumina HiSeq 2000.

\section{Sequence processing and transcriptome assembly}

Sequencing reads were filtered by removing those flagged as low quality by the Illumina software, then adapter sequences and low-quality bases were trimmed from the end of reads using the software Trimmomatic (http:// www.usadellab.org/cms/index.php?page $=$ trimmomatic). Data quality was assessed using the program FastQC (http://www.bioinformatics.babraham.ac.uk/projects/fastqc) and all of the sequence data were combined for transcriptome assembly using the program Trinity. ${ }^{35}$ The resulting unigenes were further assembled using the program iAssembler ${ }^{36}$ and then screened using the program DeconSeq ${ }^{37}$ to remove any unigenes of bacterial, viral or mammalian origin. DeconSeq threshold values for removal were greater than or equal to $95 \%$ sequence identity and sequence coverage.
Transcriptome refinement and analysis

A BLAST search ${ }^{38}$ (July 2012) was performed to compare unigenes to the NCBI non-redundant protein database with a significance level of $1.0 \times 10^{-6}$ using the blastx algorithm implemented by the program Blast2GO. ${ }^{39}$ Unigene expression estimates, measured as FPKM values, were calculated with the program RSEM ${ }^{40}$ which aligned the raw reads to the assembled unigenes. Trinity ${ }^{35}$ was used to identify unigenes containing predicted open reading frames. These results were then applied to refine the set of unigenes. As described in the Trinity developers' recommendations, the union of all transcripts with (i) significant homology to a known protein as determined through a blastx search; (ii) a predicted open reading frame; and/or (iii) an expression level, measured by the FPKM value, of greater than or equal to one were combined to remove likely assembly artifacts from the set of unigenes. To reduce the unigene set to representative transcripts, BLAST $^{38}$ was used to query the 99839 well-supported contigs against themselves. Perl scripts were written to merge self-alignments into groups likely originating from the same gene model.

Candidate coding regions within the transcripts were detected by ESTscan ${ }^{41}$ with the Arabidopsis thaliana scoring matrix. The resulting subset of transcripts was screened against the SWISS-PROT and TrEMBL ${ }^{42}$ protein databases using BLASTx with a cutoff of $1.0 \times 10^{-20}$ to assign putative functions to unigenes. Predicted peptides were analyzed using InterProScan ${ }^{43}$ to identify functional protein domains and assign Gene Ontology (GO) terms. SWISS-PROT manual curation of GO terms was also utilized to assign terms. ${ }^{44}$ Transcripts were queried against the watermelon genome assembly $\mathrm{v} 1 \mathrm{cod}$ ing sequence (watermelon_v1.cds) ${ }^{21}$ and the cucumber genome assembly v2 coding sequence (cucumber v2.cds) ${ }^{15}$ using BLASTn. Unigenes with a resulting e-value of less than $1.0 \times 10^{-20}$ were considered to have a significant match. The 55949 fruit and seed transcripts were compared to the Blanca et al. ${ }^{28}$ root, leaf and flower transcriptome by performing a reciprocal blast search of one against the other using BLASTn with a significance level of $1.0 \times 10^{-25}$. Bioconductor, using the methods of Sanchez, Salicru and Ocana ${ }^{45}$ was used to determine if there was a statistical difference in GO term representation between the unigenes unique to each transcriptome. ${ }^{46}$

\section{RESULTS AND DISCUSSION}

Transcriptome sequencing and assembly

RNA was extracted from fruit mesocarp and seed tissues of selfpollinated 'Sweet REBA' acorn squash fruit at 5, 10, 15, 20 and 40 days after pollination (Figure 1). All samples were pooled to create three biological replicates of a comprehensive fruit and seed sequencing library and paired-end reads were sequenced on one lane of an Illumina HiSeq 2000, resulting in a total of 156540465 pairs of 100 base pair (bp) reads (Table 1 ). After removing low-quality reads and trimming both adapter sequences and low-quality bases from the ends of reads, 141838600 paired reads remained, with an average length of $98.46 \mathrm{bp}$ and an average quality score of 36.98 , constituting a total of almost $28 \mathrm{Gbp}$ of sequence (reads available in the NCBI Sequence Read Archive repository, run accession numbers SRR747836, SRR747920 and SRR747922). All sequence data were combined and assembled using the program Trinity ${ }^{35}$ to yield 161 780 contigs. These contigs were further assembled using the program iAssembler, ${ }^{36}$ which resulted in a total of 150044 contigs. The contigs were then screened using the program DeconSeq ${ }^{37}$ to remove 2081 bacterial and mammalian sequence artifacts, leaving 147963 unigenes with an average length of $1311 \mathrm{bp}$.

The unigenes were filtered to remove any sequences likely resulting from possible sequencing or assembly errors by only retaining unigenes that had homology to a known protein, a predicted open reading frame and/or a FPKM expression estimate value of one or more. There were 83221 unigenes with homology to a known protein, 57598 with a predicted open reading frame, and 52374 with an FPKM expression estimate value of greater than or equal to one. These overlapping subsets of unigenes were combined into a set of 99839 unigenes, which were then clustered using self-BLAST to group alternatively-spliced transcripts originating from the same gene. An identity of $99.9 \%$ was determined to be the optimal value for merging transcripts likely transcribed from the same gene without merging putative paralogous genes. The longest unigene was 


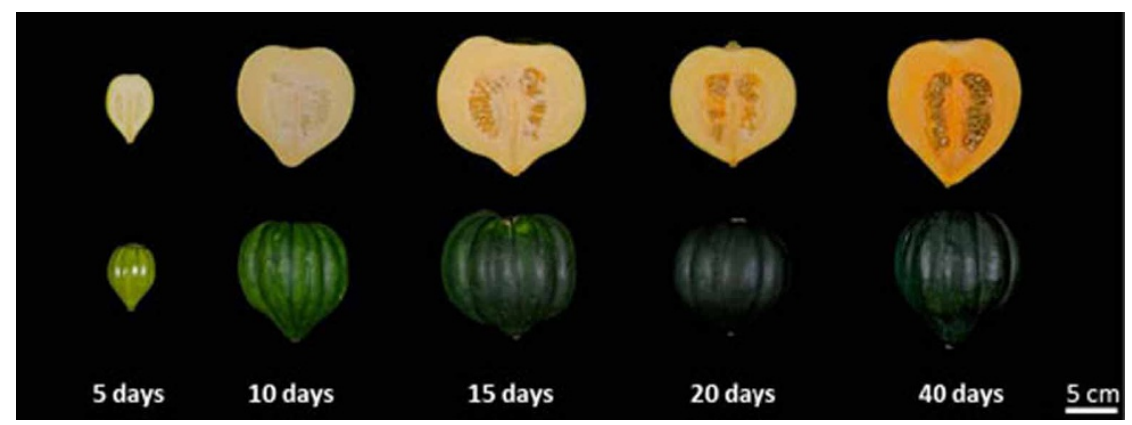

Figure 1. 'Sweet REBA' acorn squash fruit at five developmental time points. Self-pollinated fruit were harvested at 5, 10, 15, 20 and 40 days after pollinations. Photos are of the interior and exterior of representative fruit at each time point.

chosen to represent each cluster in the final transcriptome, resulting in a total of 55949 unigenes (Unigene sequences available in Supplementary File S1. This Transcriptome Shotgun Assembly project has been deposited at DDBJ/EMBL/GenBank under the accession GBZI00000000. The version described in this paper is the first version, GBZI01000000.).

Transcriptome analysis, annotation and functional characterization The final transcriptome consisted of 55949 unigenes. The unigenes ranged in length from $201 \mathrm{bp}$ to $17024 \mathrm{bp}$, with an average length of $1315 \mathrm{bp}$, a median length of $876 \mathrm{bp}$ and a total sequence length of $73559618 \mathrm{bp}$. The high average and median unigene lengths suggested that we were successful in the assembly of many fulllength transcripts, although 18561 unigenes measured 500 bp or less and were likely only partial transcripts. A BLAST search confirmed that the longest unigenes were homologous to known genes of similar length, indicating that they were likely correctly assembled. To verify that unigenes shorter than $500 \mathrm{bp}$ were indeed mainly partial transcripts, the unigene coverage of matches was considered. A total of 5921 short unigenes had significant matches to sequences in the NCBI nr database. Of these matches, the average percent of coverage of the subject sequence was $24 \%$. Only $7.6 \%$ of these unigenes matched $50 \%$ or more of the best $\mathrm{nr}$ subject, suggesting that these were truncated transcripts. With the future addition of more sequence data, it is likely that many of these short unigenes will be assembled together into full-length unigenes, reducing the total number of unigenes in the transcriptome.

A total of 41568 unigenes were predicted to have a single open reading frame and 18 unigenes were split into two open reading frames. This subset of unigenes was annotated based on homology to proteins found in SWISS-PROT and TrEMBL, with precedence given to SWISS-PROT annotations (for annotations, see Supplementary Table S1; for blast results, see Supplementary Table S2). The GenBank NR and TAIR10 databases were also queried, but results were not used in the final annotation. Of the four databases, the highest number of matches was found in GenBank NR (Table 2). Unigenes were translated into predicted proteins so that protein domains GO terms could be assigned to the predicted proteins, classifying them based on predicted involvement in biological processes, molecular functions, or cellular components. ${ }^{47}$ A total of 153 563 GO terms were assigned to $63 \%$ (26 049) of the predicted proteins (Supplementary Table S1). The number of GO terms assigned per unigene ranged from 1 to 68 for those proteins with
GO annotations, with an average of six GO terms per unigene. These GO terms fell into three categories, with $40 \%$ assigned to biological process, $21 \%$ assigned to molecular function and $39 \%$ assigned to cellular component. A range of biological processes were represented, with large numbers of unigenes assigned to categories relevant to fruit and seed development, such as 'seed development', 'embryo development', 'regulation of developmental process', 'macromolecule metabolic process', 'biosynthetic process', 'developmental growth' and 'multicellular organismal development' (Supplementary Figures S1-S3).

\section{Analysis of alternative splicing}

Approximately $44 \%$ of unigenes were collapsed by self-blast while other overlap-based means of collapsing failed, suggesting that the variants may be alternative isoforms or mis-assemblies. To assess the structure of collapsed unigenes, the clusters were further analyzed; the average number of members in a cluster was $2.5 \pm 6.8$, ranging from 1 member to 200 members (Supplementary Table S1). The clusters with the largest number of constituents were predicted to be DNA glycosylase and MYB genes. Both of these classes of genes are known to undergo alternative splicing, ${ }^{48,49}$ further reinforcing the notion that the isoforms identified were alternatively spliced transcripts and supporting our designation of these as such and their subsequent representation by the longest contig in each cluster.

Comparison with cucumber and watermelon genomes A total of 31307 unigenes had significant hits to 18381 cucumber coding sequences, representing approximately $72 \%$ of the predicted cucumber coding sequences (Supplementary Table S1). The average coding sequence coverage by the homologous unigene was $90 \%$ for cucumber coding sequence with hits. A total of 4573 unigenes with matches to a cucumber coding sequence were shorter than 500 base pairs and 4351 of these covered less than $90 \%$ of their respective match, suggesting that these unigenes are likely partial transcripts. Similarly, 33588 unigenes significantly matched 16903 watermelon coding sequences, representing $72 \%$ of the watermelon predicted coding sequences (Supplementary Table S1). The average coverage of watermelon coding sequence by squash matches was $91 \%$. A total of 4868 out of 5110 unigenes shorter than 500 bp had significant matches that covered less than $90 \%$ of their respective watermelon coding sequence match. Overall, these comparisons indicated that the majority of our unigenes were fully assembled,

Table 1. Summary of transcriptome sequencing data

\begin{tabular}{lcccc}
\hline Reads & Total number of paired reads & Average read length $(\mathrm{bp})$ & Total sequence length (bp) & Average quality score \\
\hline Raw reads & 156540465 & 100 & 31308093000 & 34.78 \\
High-quality reads & 141838600 & 98.5 & 27929628643 & 36.98 \\
\hline
\end{tabular}


Table 2. Results of squash fruit and seed transcriptome annotation based on homology

\begin{tabular}{lc}
\hline Database & Number of BLAST hits \\
\hline SWISS-PROT & $22934(55 \%)$ \\
TrEMBL & $33116(80 \%)$ \\
TAIR10 & $30590(74 \%)$ \\
GenBank NR & $34794(84 \%)$ \\
\hline
\end{tabular}

All unigenes with an open reading frame were blasted against four databases and the number of unigenes with a significant hit are reported. Blast hits were filtered by $e$-value less than $1.0 \times 10^{-20}$.

with the exception of those shorter than $500 \mathrm{bp}$, and that the transcriptome as a whole was comprehensive.

Comparison with root, leaf and flower $C$. pepo transcriptome The $C$. pepo fruit transcriptome was compared with the previously published $C$. pepo transcriptome sequenced from root, leaf and flower tissue. ${ }^{28}$ The root, leaf and flower transcriptome was assembled from sequences derived from a scallop-type and a zucchini-type squash and consisted of 49610 unigenes with an average length of $626 \mathrm{bp.}^{28}$ The fruit and seed transcriptome had a similar number of unigenes as the previous transcriptome, though it had a longer average unigene length. The greater sequencing depth of the fruit and seed transcriptome allowed by the use of Illumina sequencing technology may have enabled the assembly of more full-length transcripts.

To more closely compare the two $C$. pepo transcriptomes, we conducted a reciprocal BLAST search ${ }^{38}$ in which each transcriptome was compared to the other transcriptome using the blastn algorithm with a significance threshold of $1.0 \times 10^{-25}$ (Table 3). When the root, leaf and flower transcriptome was compared to the fruit and seed transcriptome, $96 \%$ (47 433) of the root, leaf, and flower unigenes were significantly similar to one or more of the unigenes in the fruit and seed transcriptome, with $4 \%$ not significantly similar. The matches corresponded to 22749 fruit and seed unigenes, suggesting redundancy in the root, leaf and flower transcriptome. From the reciprocal BLAST search, we found that 61\% (33 988) of the fruit and seed unigenes were significantly similar to one or more of the unigenes in the root, leaf and flower transcriptome, with 39\% not significantly similar (unigenes not significantly similar are noted in Supplementary Table S1). These matches were to 22812 of the root, leaf and flower transcripts. Additionally, 19831 unigenes had the same reciprocal match in both blast results, suggesting that these could be orthologous unigenes between zucchini and acorn squash.

To characterize the unique sequences in the fruit and seed transcriptome, their matches in the SWISS-PROT, TrEMBL, nr and TAIR10 sequence databases were assessed (e-value $\left.1.0 \times 10^{-20}\right)$. Of the unique fruit and seed unigenes, 53\% (11 671) had a predicted open reading frame and $38 \%$ ( 8315 ) had significant similarity to previously identified sequences. Therefore, our transcriptome sequencing identified more than 8000 novel unigenes in the fruit and seed

Table 3. Comparison of fruit and seed transcriptome with root, leaf and flower transcriptome

\begin{tabular}{lcc}
\hline & $\begin{array}{c}\text { Unigenes with no significant } \\
\text { similarity to other } \\
\text { transcriptome }\end{array}$ & $\begin{array}{c}\text { Unigenes with significant } \\
\text { similarity to other } \\
\text { transcriptome }\end{array}$ \\
\hline Fruit and seed & $21961(39 \%)$ & $33988(61 \%)$ \\
Root, leaf and flower & $2177(4 \%)$ & $47433(96 \%)$ \\
\hline
\end{tabular}

A reciprocal BLAST search was conducted to compare the two $C$. pepo

transcriptomes. Each transcriptome was compared to the other transcriptome using the blastn algorithm with a significance threshold of $1.0 \times 10^{-25}$.
Table 4. Carotenoid metabolism gene homologs found in fruit transcriptome

\begin{tabular}{ll}
\hline Carotenoid metabolism gene & \multicolumn{1}{c}{ C. pepo unigene homolog(s) } \\
\hline$\beta$-carotene hydroxylase & CP112262 \\
Carotenoid cleavage dioxygenase & CP134539 \\
Carotenoid isomerase & CP040968 \\
DOXP reductoisomerase & CP135419 \\
DOXP synthase & CP135924, CP060201, CP060202 \\
$\varepsilon$-hydroxylase & CP029795 \\
Geranylgeranyl hydrogenase & CP056527, CP056528, CP072215, CP072216 \\
GGPP synthase & CP135982, CP031308 \\
IPP isomerase & CP096828, CP006070, CP135789 \\
Lycopene $\beta$-cyclase & CP073600 \\
Lycopene $\varepsilon$-cyclase & CP120024 \\
Orange gene & CP135849 \\
Phytoene desaturase & CP120142, CP120144 \\
Phytoene synthase & CP005213, CP097163 \\
Violaxanthin de-epoxidase & CP068838 \\
$\zeta$-carotene desaturase & CP071571 \\
ל-carotene isomerase & CP003737 \\
Zeaxanthin epoxidase & CP041038 \\
\hline
\end{tabular}

Homologs to functionally characterized carotenoid metabolism genes were identified in the fruit and seed transcriptome through a BLAST search. The best candidate(s) for each gene are listed, identified by unigene number.

${ }^{\mathrm{a}}$ Homology found to genes annotated with only putative function.

transcriptome that had not been previously sequenced in the species. To examine gene enrichment in the two datasets, GO terms for the root, leaf and flower transcriptome were obtained from Blanca et al. ${ }^{28}$ A total of 606 unique root, leaf and flower unigenes and 5437 unique fruit and seed unigenes had at least one associated GO term. Statistically significant differences between the GO terms represented in the two samples were found for the level 2 biological process, molecular function and cellular component groups $(P<0.05)$. The unique fruit and seed unigenes were enriched for development-related GO terms, as was expected for the fruit and seed tissues sampled at five different developmental time points. These GO terms included 'cellular developmental process', 'anatomical structure morphogenesis', 'developmental maturation', 'developmental process involved in reproduction', 'seed development', 'embryo development' and 'cellular process involved in reproduction', confirming that unigenes unique to fruit and seed development were represented in our transcriptome.

Identification of fruit quality genes

Several metabolic processes that are crucial for winter squash fruit quality occur during fruit development, including the synthesis of carotenoids, sucrose and starch. To demonstrate the future utility of this transcriptome for the study of fruit quality, we identified genes involved in these processes in our transcriptome. A BLASTn search $^{38}$ was conducted to query all biosynthetic genes known to be involved in carotenoid, sugar and starch metabolism against the fruit and seed transcriptome to identify squash homologs of the genes. Squash unigenes homologous to functionally characterized genes were identified for 18 genes involved in carotenoid synthesis, storage, and degradation (Table 4), 18 genes involved in sucrose metabolism (Table 5) and 14 genes involved in starch metabolism (Table 6), demonstrating that this transcriptome fully captures gene expression related to these metabolic processes of interest.

Structural genes known to control flux or act at pathway branchpoints in carotenoid formation will be informative in future analyses of color and nutrient concentration in developing squash fruit. The carotenoid unigenes identified include the gene DOXP synthase, whose product is predicted to synthesize a regulatory step of 
Table 5. Sugar metabolism gene homologs found in fruit transcriptome

\begin{tabular}{|c|c|}
\hline Simple sugar metabolism gene & C. pepo unigene homolog(s) \\
\hline Acid $\alpha$-galactosidase & СР031132, СР041982 \\
\hline Acid invertase & СР078022, СР039349 \\
\hline Alkaline $\alpha$-galactosidase & CP135438, СР082553, СР082552, СР097985 \\
\hline Galactokinase & СР100962, СР100963 \\
\hline Galactose-1-phosphate uridyltransferase & CP115252, CP115240 \\
\hline Hexokinase & СР081918 \\
\hline Phosphofructokinase & СР076642 \\
\hline Phosphoglucomutase & CP124387, СР005960 \\
\hline Sucrose synthase & СР039490, СР036200, СР033875 \\
\hline Sucrose transporter & CP123643, CP123649, СР008293, СР009234 \\
\hline Sucrose-phosphate phosphatase & СР033610, СР078010 \\
\hline Sucrose-phosphate synthase & CP125836 \\
\hline
\end{tabular}

Homologs to functionally characterized simple sugar metabolism genes were identified in the fruit and seed transcriptome through a BLAST search. The best candidate(s) for each gene are listed, identified by unigene number.

carotenoid biosynthesis in tomato fruit. ${ }^{50}$ DOXP synthase is the first enzyme in the DOXP pathway that produces isopentenyl diphosphate, the precursor for carotenoids. ${ }^{51}$ The unigene for phytoene synthase, which performs the first committed step in carotenoid synthesis and has been shown to be rate-limiting in marigolds, canola and tomato, ${ }^{51}$ was also identified. An important branchpoint in the squash carotenoid pathway involves lycopene $\varepsilon^{-}$ cyclase and lycopene $\beta$-cyclase, also identified in the transcriptome, which both encode enzymes that act on lycopene to direct flux towards either lutein or $\beta$-carotene, ${ }^{14}$ the two primary carotenoids in C. pepo. ${ }^{2}$ Natural variation in lycopene $\varepsilon$-cyclase in maize influences the partition of carotenoids between the two branches of the pathway, ${ }^{52}$ so these genes are also likely to impact the ratio of lutein to $\beta$-carotene in squash. The orange gene (Or) was also identified, which encodes an enzyme that is important for regulating the accumulation of carotenoids through the differentiation of chromoplasts and enables high levels of carotenoid accumulation in cauliflower and potato. ${ }^{53}$

The unigenes also included key genes involved in carbohydrate metabolism. Sucrose-phosphate synthase and sucrose-phosphate phosphatase were identified, which encode enzymes that act sequentially to synthesize sucrose from UDP-glucose and fructose 6-phosphate. Sucrose-phosphate synthase is a regulatory step for sucrose biosynthesis ${ }^{54}$ and its higher enzyme activity coincided with higher sucrose accumulation in pear ${ }^{55}$ and in muskmelon. ${ }^{56}$ Starch synthesis genes identified included ADP-glucose pyrophosphorylase and AATPT (amyloplastidial ATP/ADP translocator). ADPglucose pyrophosphorylase synthesizes ADP-glucose from glucose1-phosphate and is the first committed step in starch biosynthesis. ${ }^{57}$ AATPT transfers ATP and ADP between the cytosol and the amyloplast, providing the ATP needed for starch biosynthesis. ${ }^{57}$ Also identified was a unigene encoding $\alpha$-amylase, which is the primary enzyme in squash that breaks down starch, yielding simple sugars that confer sweetness. ${ }^{58}$ Together, these genes form the core of hypotheses that will be tested in future comparative studies of winter squash quality.

\section{CONCLUSION}

In this study, we report the first Cucurbita pepo winter squash fruit and seed transcriptome, with more than 141 million high-quality paired-end sequencing reads compiled over five developmental

Table 6. Starch metabolism gene homologs found in fruit transcriptome

\begin{tabular}{ll}
\hline Starch metabolism gene & \multicolumn{1}{c}{ C. pepo unigene homolog(s) } \\
\hline ADP-glucose pyrophosphorylase L & CP101035, CP101034 \\
ADP-glucose pyrophosphorylase S & CP126587 \\
$\alpha$-amylase & CP135677 \\
$\alpha$-glucosidase & $\mathrm{CP} 144486, \mathrm{CP} 116493, \mathrm{CP} 116492, \mathrm{CP} 006281$ \\
Amyloplastidial ATP/ADP translocator & $\mathrm{CP} 098764, \mathrm{CP} 001903, \mathrm{CP} 098765, \mathrm{CP} 001904$ \\
$\beta$-amylase & $\mathrm{CP} 070598, \mathrm{CP} 070601, \mathrm{CP} 070602, \mathrm{CP} 074740$ \\
Cell wall invertase & $\mathrm{CP} 052558$ \\
debranching enzyme & $\mathrm{CP} 039826$ \\
Glucose phosphate transporter & $\mathrm{CP} 086414, \mathrm{CP} 081420, \mathrm{CP} 034210, \mathrm{CP} 034209, \mathrm{CP} 037833$ \\
Phosphoglucose isomerase & $\mathrm{CP} 109433$ \\
Starch phosphorylase & $\mathrm{CP} 033924, \mathrm{CP} 004861, \mathrm{CP} 136313$ \\
Starch synthase-granule bound & $\mathrm{CP} 118399, \mathrm{CP} 118397, \mathrm{CP} 118398, \mathrm{CP} 118396$ \\
Starch synthase-soluble & $\mathrm{CP} 113496, \mathrm{CP} 113492$ \\
Starch-branching enzyme & $\mathrm{CP} 033816, \mathrm{CP} 120408, \mathrm{CP} 120407$ \\
\hline
\end{tabular}

Homologs to functionally characterized starch metabolism genes were identified in the fruit and seed transcriptome through a BLAST search. The best candidate(s) for each gene are listed, identified by unigene number. 
time points and assembled into a final transcriptome of 55949 unigenes. Approximately $85 \%$ of the unigenes with open reading frames shared homology with known proteins and $62 \%$ could be functionally annotated. This transcriptome was compared to the cucumber and watermelon genomes, as well as the previously sequenced C. pepo root, leaf and flower transcriptome, which confirmed that it was comprehensive, had a majority of full-length unigenes, and contained unigenes unique to fruit and seed development. This fruit and seed transcriptome represents a major contribution to C. pepo genomic resources, with more than 8000 C. pepo unigenes homologous to known genes that are new to the sequenced exome and will be useful in future genome annotation efforts. Further, the identification of likely candidates for carotenoid and carbohydrate metabolism genes suggests that this novel resource will enable further study of fruit quality and development to enhance future squash breeding efforts that seek to produce higher quality fruit with greater nutritional and culinary value.

\section{COMPETING INTERESTS}

The authors declare no conflict of interest.

\section{ACKNOWLEDGEMENTS}

We thank the Cornell University Biotechnology Resource Center and Giovanna Danies for bioinformatics support, Li Li and Zhangjun Fei for experimental design advice, Scott Anthony for providing plant care and Paige Roosa for comments on the manuscript. This research was supported through funds from Cornell University and the Vegetable Breeding Institute. Support for Lindsay Wyatt was provided by a Cornell University Presidential Life Sciences Fellowship, USDA National Needs Graduate Fellowship Competitive Grant No. 2008-38420-04755 from the National Institute of Food and Agriculture, and the Agriculture and Food Research Initiative Competitive Grant No. 2013-67011-21122 from the USDA National Institute of Food and Agriculture.

\section{REFERENCES}

1 Ferriol M, Picó B. Pumpkin and winter squash. In: Prohens J, Nuez F (ed.) Vegetables I. Vol. 1. New York: Springer, 2008: 317-349.

2 Azevedo-Meleiro CH, Rodriguez-Amaya DB. Qualitative and quantitative differences in carotenoid composition among Cucurbita moschata, Cucurbita maxima, and Cucurbita pepo. J Agric Food Chem 2007; 55: 4027-4033.

3 Cazzonelli $\mathrm{Cl}$, Pogson BJ. Source to sink: regulation of carotenoid biosynthesis in plants. Trends Plant Sci 2010; 15: 266-274.

4 Idouraine A, Kohlhepp EA, Weber CW, Warid WA, MartinezTellez JJ. Nutrient constituents from eight lines of naked seed squash (Cucurbita pepo L). J Agric Food Chem 1996; 44: 721-724.

5 Murkovic M, Mulleder U, Neunteufl $\mathrm{H}$. Carotenoid content in different varieties of pumpkins. J Food Compos Anal 2002; 15: 633-638.

6 Yoshida H, Shougaki Y, Hirakawa Y, Tomiyama Y, Mizushina Y. Lipid classes, fatty acid composition and triacylglycerol molecular species in the kernels of pumpkin (Cucurbita spp.) seeds. J Sci Food Agric 2004; 84: 158-163.

7 Robinson RW, Decker-Walters DS. Cucurbits. New York: CAB International, 1997.

8 Gajewski M, Radzanowska J, Danilcenko H, Jariene E, Cerniauskiene J. Quality of pumpkin cultivars in relation to sensory characteristics. Not Bot Hort Agrobot Cluj 2008; 36: 73-79.

9 Itle RA, Kabelka EA. Correlation between $L * a * b *$ color space values and carotenoid content in pumpkins and squash (Cucurbita spp.). HortScience 2009; 44: 633-637.

10 Cumarasamy R, Corrigan V, Hurst P, Bendall M. Cultivar differences in New Zealand "Kabocha" (buttercup squash, Cucurbita maxima). NZ J Crop Hort Sci 2002; 30: 197208.

11 Corrigan VK, Hurst PL, Potter JF. Winter squash (Cucurbita maxima) texture: sensory, chemical, and physical measures. NZ J Crop Hort Sci 2001; 29: 111-124.

12 Hurst PL, Corrigan VK, Koolaard J. Genetic analysis of sweetness and textura attributes in winter squash (Cucurbita maxima). NZ J Crop Hort Sci 2006; 34: 359-367.

13 Irving DE, Hurst PL, Ragg JS. Changes in carbohydrates and carbohydrate metabolizing enzymes during the development, maturation, and ripening of buttercup squash (Cucurbita maxima D 'Delica'). J Am Soc Hort Sci 1997; 122: 310-314.

14 Lu S, Li L. Carotenoid metabolism: biosynthesis, regulation, and beyond. J Integr Plant Biol 2008; 50: 778-785.

15 Huang SW, Li RQ, Zhang ZH et al. The genome of the cucumber, Cucumis sativus L. Nat Genet 2009; 41: 1275-1281.
16 Clepet $C$, Joobeur T, Zheng Y et al. Analysis of expressed sequence tags generated from full-length enriched CDNA libraries of melon. BMC Genomics 2011; 12: 1-12.

17 Diaz A, Fergany M, Formisano $G$ et al. A consensus linkage map for molecular markers and Quantitative Trait Loci associated with economically important traits in melon (Cucumis melo L.). BMC Plant Biol 2011; 11: 1-14.

18 Garcia-Mas J, Benjak A, Sanseverino W et al. The genome of melon (Cucumis melo L.). Proc Natl Acad Sci USA 2012; 109: 11872-11877.

19 Ren Y, Zhao H, Kou Q et al. A high resolution genetic map anchoring scaffolds of the sequenced watermelon genome. PLoS One 2012; 7: e29453.

20 Zhang WW, Pan JS, He HL et al. Construction of a high density integrated genetic map for cucumber (Cucumis sativus L.). Theor Appl Genet 2012; 124: 249-259.

21 Guo SG, Zhang JG, Sun HH et al. The draft genome of watermelon (Citrullus lanatus) and resequencing of 20 diverse accessions. Nat Genet 2013; 45: 51-58.

22 Arumuganathan K, Earle ED. Nuclear DNA content of some important plant species. Plant Mol Biol Rep 1991; 9: 208-218.

23 Weeden F. Isozyme studies indicate that the genus Cucurbita is an ancient tetraploid. Cucurbit Genet Cooper Rep 1984; 7: 84-85.

24 Zraidi A, Stift G, Pachner M, Shojaeiyan A, Gong L, Lelley T. A consensus map for Cucurbita pepo. Mol Breeding 2007; 20: 375-388.

25 Gong L, Stift G, Kofler R, Pachner M, Lelley T. Microsatellites for the genus Cucurbita and an SSR-based genetic linkage map of Cucurbita pepo L. Theor Appl Genet 2008; 117: 37-48.

26 Gong L, Pachner M, Kalai K, Lelley T. SSR-based genetic linkage map of Cucurbita moschata and its synteny with Cucurbita pepo. Genome 2008; 51: 878-887.

27 Esteras C, Gomez P, Monforte AJ et al. High-throughput SNP genotyping in Cucurbita pepo for map construction and quantitative trait loci mapping. BMC Genomics 2012; 13: 1-21.

28 Blanca J, Canizares J, Roig C, Ziarsolo P, Nuez F, Pico B. Transcriptome characterization and high throughput SSRs and SNPs discovery in Cucurbita pepo (Cucurbitaceae). BMC Genomics 2011; 12: 1-15.

29 Wu T, Luo S, Wang R et al. The first Illumina-based de novo transcriptome sequencing and analysis of pumpkin (Cucurbita moschata Duch.) and SSR marker development. Mol Breeding 2014; 34: 1437-1447.

30 Ando K, Grumet R. Transcriptional profiling of rapidly growing cucumber fruit by 454-pyrosequencing analysis. J Am Soc Hort Sci 2010; 135: 291-302.

31 Ando K, Carr KM, Grumet R. Transcriptome analyses of early cucumber fruit growth identifies distinct gene modules associated with phases of development. BMC Genomics 2012; 13: 1-16.

32 Zenoni S, Ferrarini A, Giacomelli E et al. Characterization of transcriptional complexity during berry development in Vitis vinifera using RNA-Seq. Plant Physiol 2010; 152: 1787-1795.

33 Rohrmann J, Tohge T, Alba R et al. Combined transcription factor profiling microarray analysis and metabolite profiling reveals the transcriptional control of metabolic shifts occurring during tomato fruit development. Plant $J$ 2011; 68: 999-1013.

34 Loy JB. Morpho-physiological aspects of productivity and quality in squash and pumpkins (Cucurbita spp.). Crit Rev Plant Sci 2004; 23: 337-363.

35 Grabherr MG, Haas BJ, Yassour M et al. Full-length transcriptome assembly from RNA-Seq data without a reference genome. Nat Biotechnol 2011; 29: 644-652.

36 Zheng Y, Zhao LJ, Gao JP, Fei ZJ. iAssembler: a package for de novo assembly of Roche-454/Sanger transcriptome sequences. BMC Bioinformatics 2011; 12: 1-8.

37 Schmieder R, Edwards R. Fast identification and removal of sequence contamination from genomic and metagenomic datasets. PLoS One 2011; 6: e17288.

38 Altschul SF, Madden TL, Schaffer AA et al. Gapped BLAST and PSI-BLAST: a new generation of protein database search programs. Nucleic Acids Res 1997; 25: 33893402.

39 Conesa A, Gotz S, Garcia-Gomez JM, Terol J, Talon M, Robles M. Blast2GO: a universal tool for annotation, visualization and analysis in functional genomics research. Bioinformatics 2005; 21: 3674-3676.

40 Li B, Dewey CN. RSEM: accurate transcript quantification from RNA-Seq data with or without a reference genome. BMC Bioinformatics 2011; 12: 1-16.

41 Iseli C, Jongeneel C, Bucher P. ESTScan: a program for detecting, evaluating, and reconstructing potential coding regions in EST sequences. Proc Int Conf Intell Syst Mol Biol. 1999; 1999: 138-148.

42 Apweiler R, Martin MJ, O'Donovan C et al. Update on activities at the Universal Protein Resource (UniProt) in 2013. Nucleic Acids Res 2013; 41: D43-D47.

43 Zdobnov EM, Apweiler R. InterProScan-an integration platform for the signaturerecognition methods in InterPro. Bioinformatics 2001; 17: 847-848.

44 Camon E, Magrane M, Barrell D et al. The gene ontology annotation (GOA) project: Implementation of GO in SWISS-PROT, TrEMBL, and InterPro. Genome Res 2003; 13: 662-672.

45 Sanchez A, Salicru M, Ocana J. Statistical methods for the analysis of highthroughput data based on functional profiles derived from the Gene Ontology. J Stat Plann Infer 2007; 137: 3975-3989. 
46 Carlson M, Falcon S, Pages H, Li N. GO.db: a set of annotation maps describing the entire Gene Ontology. $\mathrm{R}$ package version $2.6 .1 \mathrm{ed}$.

47 Ashburner M, Ball CA, Blake JA et al. Gene Ontology: tool for the unification of biology. Nat Genet 2000; 25: 25-29.

48 Murphy TM, Gao M. Multiple forms of formamidopyrimidine-DNA glycosylase produced by alternative splicing in Arabidopsis thaliana. J Photochem Photobiol B-Biol 2001; 61: 87-93.

49 Li JG, Li XJ, Guo L et al. A subgroup of MYB transcription factor genes undergoes highly conserved alternative splicing in Arabidopsis and rice. J Exp Botany 2006; 57: 1263-1273.

50 Lois LM, Rodriguez-Concepcion M, Gallego F, Campos N, Boronat A. Carotenoid biosynthesis during tomato fruit development: regulatory role of 1-deoxy-Dxylulose 5-phosphate synthase. Plant J 2000; 22: 503-513.

51 Hirschberg J. Carotenoid biosynthesis in flowering plants. Curr Opin Plant Biol 2001; 4: $210-218$.

52 Harjes CE, Rocheford TR, Bai L et al. Natural genetic variation in lycopene epsilon cyclase tapped for maize biofortification. Science 2008; 319: 330-333.

53 Lu S, van Eck J, Zhou X et al. The cauliflower or gene encodes a DnaJ cysteine-rich domain-containing protein that mediates high levels of beta-carotene accumulation. Plant Cell 2006; 18: 3594-3605.
54 Huber SC, Huber JL. Role and regulation of sucrose-phosphate synthase in higher plants. Annu Rev Plant Physiol Plant Mol Biol 1996; 47: 431-444.

55 Zhang HP, Wu JY, Qin GH et al. The role of sucrose-metabolizing enzymes in pear fruit that differ in sucrose accumulation. Acta Physiol Plant 2014; 36: 71-77.

56 Hubbard NL, Huber SC, Pharr DM. Sucrose phosphate synthase and acid invertase as determinants of sucrose concentration in developing muskmelon (Cucumis melo L.) fruits. Plant Physiol 1989; 91: 1527-1534.

57 Geigenberger P, Stitt M, Fernie AR. Metabolic control analysis and regulation of the conversion of sucrose to starch in growing potato tubers. Plant Cell Environ 2004; 27: 655-673.

58 Irving DE, Shingleton GJ, Hurst PL. Starch degradation in buttercup squash (I). J Am Soc Hort Sci 1999; 124: 587-590.

(c) (i) $(-)$ This work is licensed under a Creative Commons Attribution-

cc. NonCommercial-NoDerivs 3.0 Unported License. The images or other third party material in this article are included in the article's Creative Commons license, unless indicated otherwise in the credit line; if the material is not included under the Creative Commons license, users will need to obtain permission from the license holder to reproduce the material. To view a copy of this license, visit http:// creativecommons.org/licenses/by-nc-nd/3.0/

Supplemental Information for this article can be found on the Horticulture Research website (http://www.nature.com/hortres). 